\title{
BMJ Open Comparison of Hospital-at-Home models: a systematic review of reviews
}

\author{
Man Qing Leong (D) , ${ }^{1}$ Cher Wee Lim, ${ }^{2,3}$ Yi Feng Lai (i) ${ }^{2,4,5,6}$
}

To cite: Leong MQ, Lim CW, Lai YF. Comparison of Hospitalat-Home models: a systematic review of reviews. BMJ Open 2021;11:e043285. doi:10.1136/ bmjopen-2020-043285

- Prepublication history and additional material for this paper is available online. To view these files, please visit the journal online (http://dx.doi.org/10. 1136/bmjopen-2020-043285).

Received 31 July 2020 Revised 28 December 2020 Accepted 15 January 2021

Check for updates

(C) Author(s) (or their employer(s)) 2021. Re-use permitted under CC BY-NC. No commercial re-use. See rights and permissions. Published by BMJ.

${ }^{1}$ Division of Organisation Planning and Performance, Singapore General Hospital, Singapore

${ }^{2}$ Office for Healthcare

Transformation, Ministry of Health, Singapore

${ }^{3}$ Saw Swee Hock School of Public Health, National University of Singapore,

Singapore

${ }^{4}$ Department of Pharmacy,

Alexandra Hospital, Singapore

${ }^{5}$ Department of Pharmacy,

Faculty of Science, National

University of Singapore,

Singapore

${ }^{6}$ School of Public Health, University of Illinois, Chicago, Illinois, USA

Correspondence to

Man Qing Leong;

leongmanqing@gmail.com

\section{ABSTRACT}

Objectives To provide an overview of the safety and effectiveness of Hospital-at-Home (HaH) according to programme type (early-supported discharge (ESD) vs admission avoidance (AA)), and identify the model with higher evidence for addressing clinical, length of stay (LOS) and cost outcomes.

Methods A systematic review of reviews was conducted by performing a search on PubMed, EMBASE, Cochrane Database of Systematic Reviews, Web of Science and Scopus (January 2005 to June 2020) for Englishlanguage systematic reviews evaluating $\mathrm{HaH}$. Data on primary outcomes (mortality, readmissions, costs, LOS), secondary outcomes (patient/caregiver outcomes) and process indicators were extracted. Quality of the reviews was assessed using Assessment of Multiple Systematic Reviews-2. There was no registered protocol.

Results Ten systematic reviews were identified (four high quality, five moderate quality and one low quality). The reviews were classified according to three use cases. ESD reviews generally revealed comparable mortality (RR 0.92-1.03) and readmissions (RR 1.09-1.25) to inpatient care, shorter hospital LOS (MD -6.76 to -4.44 days) and unclear findings for costs. AA reviews observed a trend towards lower mortality (RR $0.77,95 \% \mathrm{Cl} 0.54$ to 1.09 ) and costs, and comparable or lower readmissions (RR 0.68-0.98). Among reviews including both programme types (ESD/AA), chronic obstructive pulmonary disease reviews revealed lower mortality (RR 0.65-0.68) and post$\mathrm{HaH}$ readmissions (RR $0.74-0.76$ ) but unclear findings for resource use.

Conclusion For suitable patients, HaH generally results in similar or improved clinical outcomes compared with inpatient treatment, and warrants greater attention in health systems facing capacity constraints and rising costs. Preliminary comparisons suggest prioritisation of AA models over ESD due to potential benefits in costs and clinical outcomes. Nonetheless, future research should clarify costs of $\mathrm{HaH}$ programmes given the current low-quality evidence, as well as address evidence gaps pertaining to caregiver outcomes and adverse events under $\mathrm{HaH}$ care.

\section{INTRODUCTION}

Healthcare systems around the world are grappling with ageing populations, increasing healthcare needs and expenditures, and shortages of hospital beds and manpower. ${ }^{12}$ Such capacity constraints decrease the efficiency and quality of care delivery, and can
Strengths and limitations of this study

- The first systematic review of reviews providing a comprehensive overview of the safety and efficacy of Hospital-at-Home $(\mathrm{HaH})$ according to programme type.

- Compares two major HaH programme types to offer relevant recommendations for health systems facing capacity constraints and rising costs.

- Highlights evidence gaps pertaining to outcomes and process indicators in the current HaH literature.

- Strength of evidence for comparison between $\mathrm{HaH}$ models is low due to heterogeneity in implementation and patient groups across studies.

lead to delays in elective and emergency treatment, ${ }^{3}$ increased mortality risk, ${ }^{45}$ lower patient and staff satisfaction, and higher costs for both patients and the health service. ${ }^{6}$ Chronic morbidity in ageing populations have further driven the rise in healthcare costs. ${ }^{7}$ These complex challenges pose a threat to the sustainability and quality of care and underscore the need to explore innovative solutions.

An alternative model of care that has been identified as a possible solution is the Hospital-at-Home $(\mathrm{HaH})$ model, in which healthcare professionals deliver hospital-level care in patients' homes for a limited period of time. ${ }^{8}$ There are two main types of $\mathrm{HaH}$ programmes-early supported discharge (ESD) and admission avoidance (AA). ESD aims to accelerate the discharge of admitted patients, thus, partially substituting hospital care. AA directly admits patients into $\mathrm{HaH}$ based on general practitioner referralsthereby avoiding physical contact with the hospital, or through direct admissions from the emergency room without inpatient stay.

One of the initial impetuses behind the $\mathrm{HaH}$ model was to reduce adverse events associated with the hospitalisation of older patients. ${ }^{9}$ Individual studies have demonstrated the benefits of $\mathrm{HaH}$ programmes in terms of safety, ${ }^{9}{ }^{10}$ effectiveness, ${ }^{11} 12$ reduced iatrogenic complications ${ }^{13}$ and cost savings. ${ }^{1415}$ While several systematic reviews 
have also been conducted, evidence for some outcomes remains unclear or conflicting, partly due to different approaches between reviews. Greater clarity may be attained by consolidating the reviews. ${ }^{16}$

In addition, despite past suggestions that outcomes such as costs and clinical complications vary between ESD and AA models due to differing extents of inpatient care substitution, ${ }^{17-19}$ these have not been comprehensively assessed. A 2017 National Institute for Health and Care Excellence guideline ${ }^{17}$ presented analyses according to programme type, but the further stratification of results based on level of care precluded any clear conclusions.

Hence, the objectives of this review were to synthesise the evidence for safety and effectiveness of $\mathrm{HaH}$ according to programme type, and assess which model had higher evidence for addressing clinical, length of stay (LOS) and cost outcomes. This would guide prioritisation in service planning, particularly for health systems that are grappling with capacity constraints and increasing costs. Process indicators and facilitators and barriers to implementation were also summarised. These are pertinent for potential adopter organisations to evaluate the feasibility of $\mathrm{HaH}$ in their respective settings as past studies have pointed to constraints in the $\mathrm{HaH}$ model (eg, in terms of patient eligibility due to medical condition severity ${ }^{20}$ and caregiver availability. ${ }^{21}$ Moreover, process indicators are valuable in assessing the fidelity of implementation and identifying components that increase the success of $\mathrm{HaH}$ implementation. ${ }^{22}$

\section{METHODS}

\section{Data sources and searches}

A search was performed on 14 January 2020 using PubMed, EMBASE, Cochrane Database of Systematic Reviews, Web of Science and Scopus. Search terms and criteria for inclusion/exclusion were determined a priori by all authors. Test searches were carried out and results were reviewed and refined by two authors before finalising the search strategy. The search was restricted to systematic reviews published after January 2005. While $\mathrm{HaH}$ pilot studies have been implemented before that, the care model took time to mature and was largely inconsistently defined prior to a seminal paper in $2005 .{ }^{9}$ Hence, reviews after 2005 were considered more representative of the $\mathrm{HaH}$ literature. The search strategy employed terms that operationalised 'review' and were related to 'HaH' (table 1). A prespecified update of the search was performed on 8 June 2020 . There was no registered protocol for this study.

\section{Study selection}

We included systematic reviews of $\mathrm{HaH}$ interventions, with $\mathrm{HaH}$ defined as the active provision of care by healthcare professionals in patients' homes for acute and post-acute conditions that otherwise would require hospital inpatient care. While $\mathrm{HaH}$ is commonly defined as the provision of acute care at home, we aligned our operational definition with Cochrane reviews which have included trials providing both acute and post-acute services, ${ }^{23} 24$ or postacute care alone. ${ }^{25-27}$ In addition, care services should only be provided for a time-limited period. ${ }^{8}{ }^{28}$ Systematic reviews including other interventions were eligible if subgroup analyses for $\mathrm{HaH}$ were presented. We included reviews which reported at least one of the following: clinical outcomes, resource use or process indicators. Process indicators were defined as measures of the processes involved in achieving programme objectives. ${ }^{29}$ English-language systematic reviews of all study designs were eligible.

Reviews of interventions that were delivered in outpatient settings, provided long-term care, or primarily involved patient self-care at home were excluded. We excluded reviews of palliative, obstetric, paediatric and mental health $\mathrm{HaH}$ schemes due to differences in these groups' care needs and objectives from general medicine patients. We excluded reviews that were entirely overlapped by a subsequent update or review of higher quality, as determined using the Assessment of Multiple Systematic Reviews-2 (AMSTAR-2).$^{30}$

Literature search and shortlist of the articles were done consistently by one investigator, and shortlisted articles were reviewed by two authors for final inclusion.

\section{Data extraction and quality assessment}

Review characteristics and relevant outcomes were extracted from the reported findings and tables in the reviews. Primary outcomes of interest were mortality, readmissions, costs and LOS. As the entire treatment duration under $\mathrm{HaH}$ is considered inpatient stay, LOS in ESD schemes includes both the stay in the hospital and home. For AA schemes, LOS refers to LOS in the home before discharge from $\mathrm{HaH}$. Secondary outcomes of interest were patient and caregiver satisfaction and caregiver burden. Process indicators (recruitment rates, adverse events) and facilitators and barriers to implementation were also extracted.

Methodological quality of the reviews was assessed using AMSTAR-2, ${ }^{30}$ which provides overall ratings (high, moderate, low, critically low) based on weaknesses in critical domains. In line with Cochrane guidelines, ${ }^{31}$ methodological quality of the primary studies was determined using the review authors' original assessments of risk of bias.

\section{Data synthesis and analysis}

Findings from included reviews were grouped, narratively summarised and compared. Due to heterogeneity between studies, a meta-analysis was not possible.

\section{Patient involvement}

Patients or the public were not involved in this research. Ethics review was not sought and patient consent was not required.

\section{RESULTS}

The search returned 7869 potentially relevant references. No relevant papers were identified from the 
Table 1 Search syntax for database search

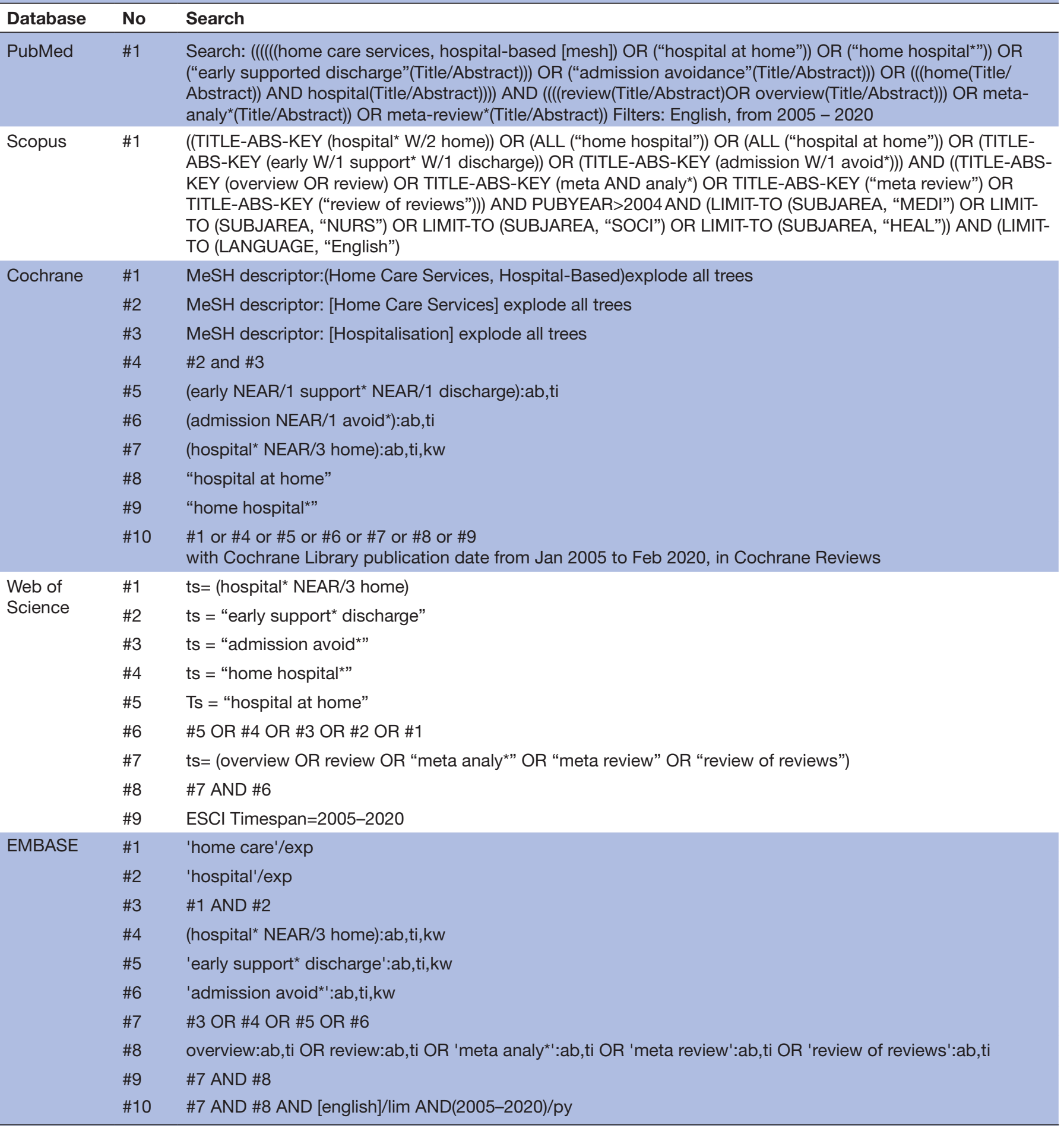

search update. Ten reviews were eventually included. The Preferred Reporting Items for Systematic Reviews and Meta-Analyses flow diagram in figure 1 details the article selection process.

\section{Review characteristics}

The ten reviews (four Cochrane reviews, six non-Cochrane reviews) were published between 2012 and 2017. Cumulatively, there were 100 relevant primary studies, with substantial overlap in studies between reviews (40\%). The studies were published between 1976 and 2016, and were mostly from the UK, US, Australia, Italy and Spain.

Some reviews either assessed only ESD or AA interventions, while several reviews included both ESD and AA studies. The reviews were categorised according to three use cases: ESD, ${ }^{28}{ }^{32} \mathrm{AA}^{83334}$ and ESD/AA. ${ }^{35-39}$ Characteristics of the reviews are summarised in table 2. Service structure of the $\mathrm{HaH}$ programmes in the primary studies varied both within and between reviews. There was 


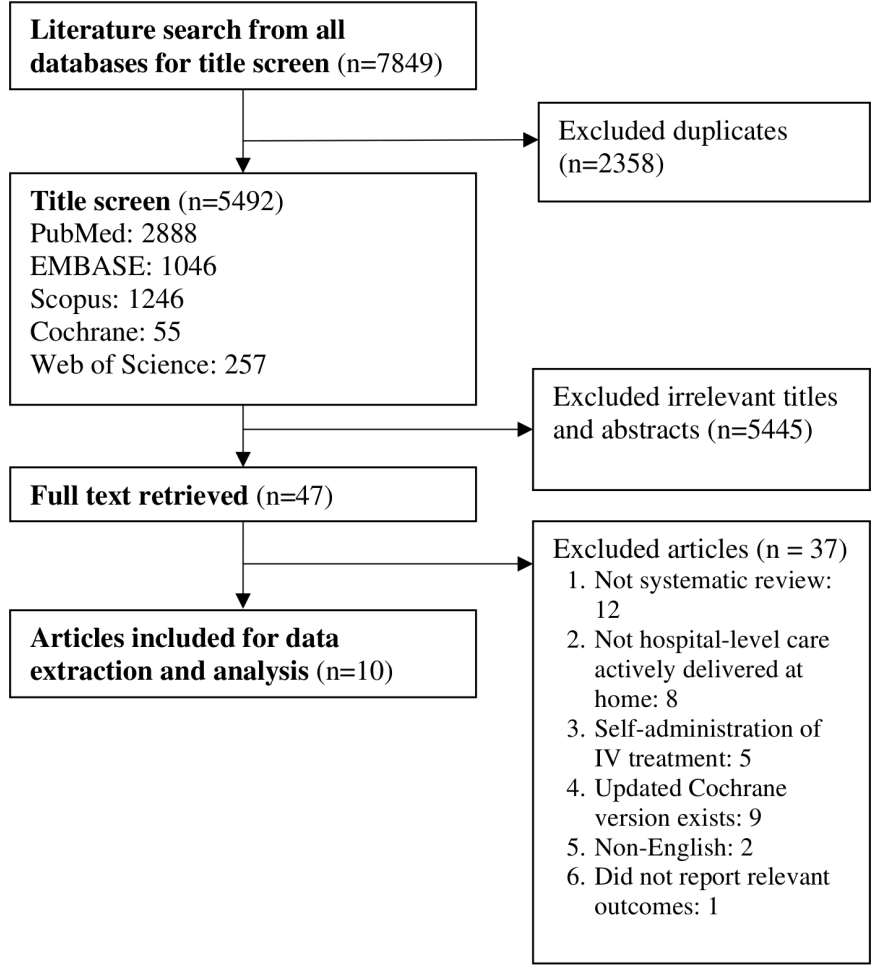

Figure 1 PRISMA flow diagram for article selection. PRISMA, Preferred Reporting Items for Systematic Reviews and Meta-Analyses.

heterogeneity in the team coordinating care (eg, hospital vs community-based), care team composition (eg, nurses, physiotherapists, occupational therapists), programme components (eg, additional elements such as patient and caregiver education), number of home visits, availability of after-hours support and specific medical services provided (eg, home oxygen, intravenous fluids).

\section{Quality of studies}

There were four reviews ${ }^{82} 3237$ of high quality, five e $^{33-3638}$ of moderate quality and one ${ }^{39}$ of low quality. Main areas of weakness were the lack of a registered protocol and failure to provide a list of excluded studies (refer to online supplemental table 1) for AMSTAR-2 ratings).

The randomised trials were mostly assessed to be at low risk of bias, while the observational studies were of moderate to high risk of bias.

\section{Outcomes}

Detailed findings and summary measures are reported in table 3. As a meta-analysis was not conducted, effect estimates across reviews were summarised descriptively in the main text where feasible to do so (ie, when the same type of effect measure was used across summarised reviews). CIs were included if the effect measure was only reported by one review.

\section{Early-supported discharge}

Clinical outcomes

Two reviews ${ }^{28} 32$ reported that ESD did not significantly affect the mortality rates of elderly patients with a mix of conditions (RR $1.03,95 \%$ CI 0.76 to 1.49 ; moderatequality evidence), patients who had a stroke (moderatequality evidence), and elective surgical patients (MD $-3.8 \%$ to $0.1 \%$; low-quality evidence).

One review ${ }^{28}$ revealed a trend towards higher readmissions in ESD schemes for a mix of conditions (RR 1.25, 95\% CI 0.98 to 1.58 ; moderate-quality evidence), whereas readmissions did not differ significantly between ESD and inpatient groups for stroke (RR 1.09, 95\% CI 0.71 to 1.66; low-quality evidence) and surgical patients (low-quality evidence). It was unclear if these analyses included readmissions during ESD care.

\section{Resource use and costs}

Two reviews ${ }^{28}{ }^{32}$ concluded that ESD significantly reduced hospital LOS for all patient groups (MD -6.76 to -4.44 days; moderate-quality evidence), although high heterogeneity in results was observed. Gonçalves-Bradley et $a l^{28}$ observed a significant increase in total LOS (in the hospital and home) across three trials for a mix of conditions (MD 6.43 days, $95 \%$ CI 2.84 to 10.03).

Two reviews ${ }^{28} 32$ found conflicting findings for the effect of ESD on costs (very low-quality evidence). High heterogeneity in cost estimates was also observed due to differences in intervention, cost components and cost measurements across trials. Only one trial factored in community costs; mean hospital cost savings under ESD (at 6 months postrandomisation) were reduced from $\$ \mathrm{~A} 4678$ (95\% CI $\$ \mathrm{~A} 2676$ to $\$ \mathrm{~A} 6680$ ) to $\$ \mathrm{~A} 2013$ (95\% CI $\$$ A669 to \$A4696) on factoring in those costs.

\section{Patient and caregiver outcomes}

Two reviews ${ }^{28} 32$ reported that ESD generally led to similar or improved levels of patient and caregiver satisfaction and caregiver strain when compared with inpatient care (low-quality evidence). However, for surgical patients, three out of five trials observed lower caregiver satisfaction under ESD. ${ }^{28}$

\section{Process indicators}

Recruitment rates were low for all patient groups, with a median of $20 \%-33 \%$ of patients meeting eligibility criteria. ${ }^{28}{ }^{32}$ Regarding adverse events and clinical complications, one review ${ }^{28}$ reported a lack of difference between ESD and inpatient groups in three trials for surgical patients, but significantly lower rates of delirium in the ESD group in a trial for a mix of conditions.

\section{Admission avoidance Clinical outcomes}

AA may reduce mortality rates compared with inpatient care. A Cochrane review ${ }^{8}$ for a mix of conditions reported a trend towards lower mortality under AA schemes at 3 months (RR $0.77,95 \%$ CI 0.54 to 1.09 ; moderate-quality evidence) and a significant reduction at 6 months (RR $0.77,95 \%$ CI 0.60 to 0.99 ; moderate-quality evidence). Two other reviews ${ }^{33} 34$ did not find significant effects on mortality (RR $0.94 ; 95 \%$ CI 0.67 to 1.32 ),$^{33}$ but raised the issue of under-powering. 


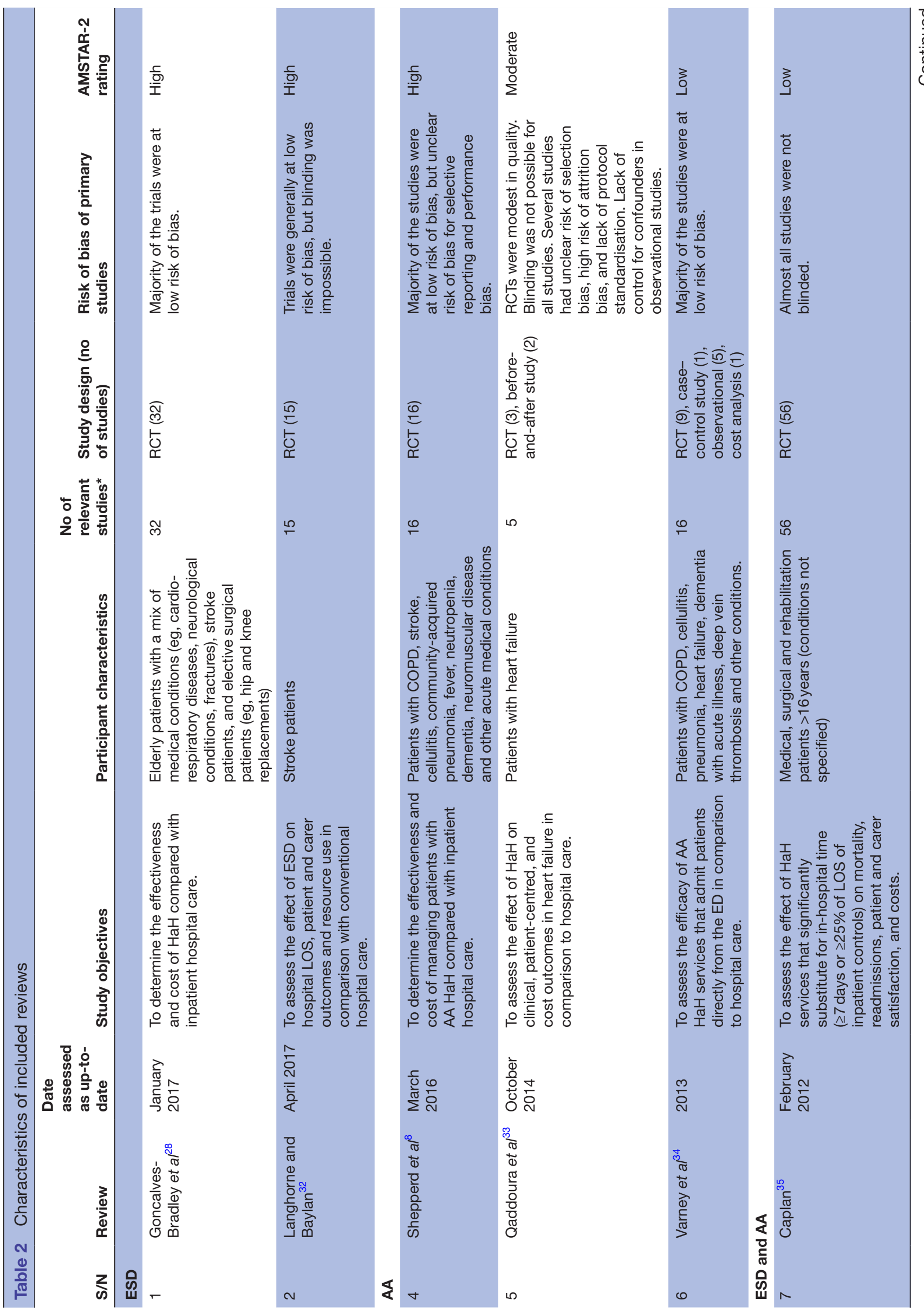




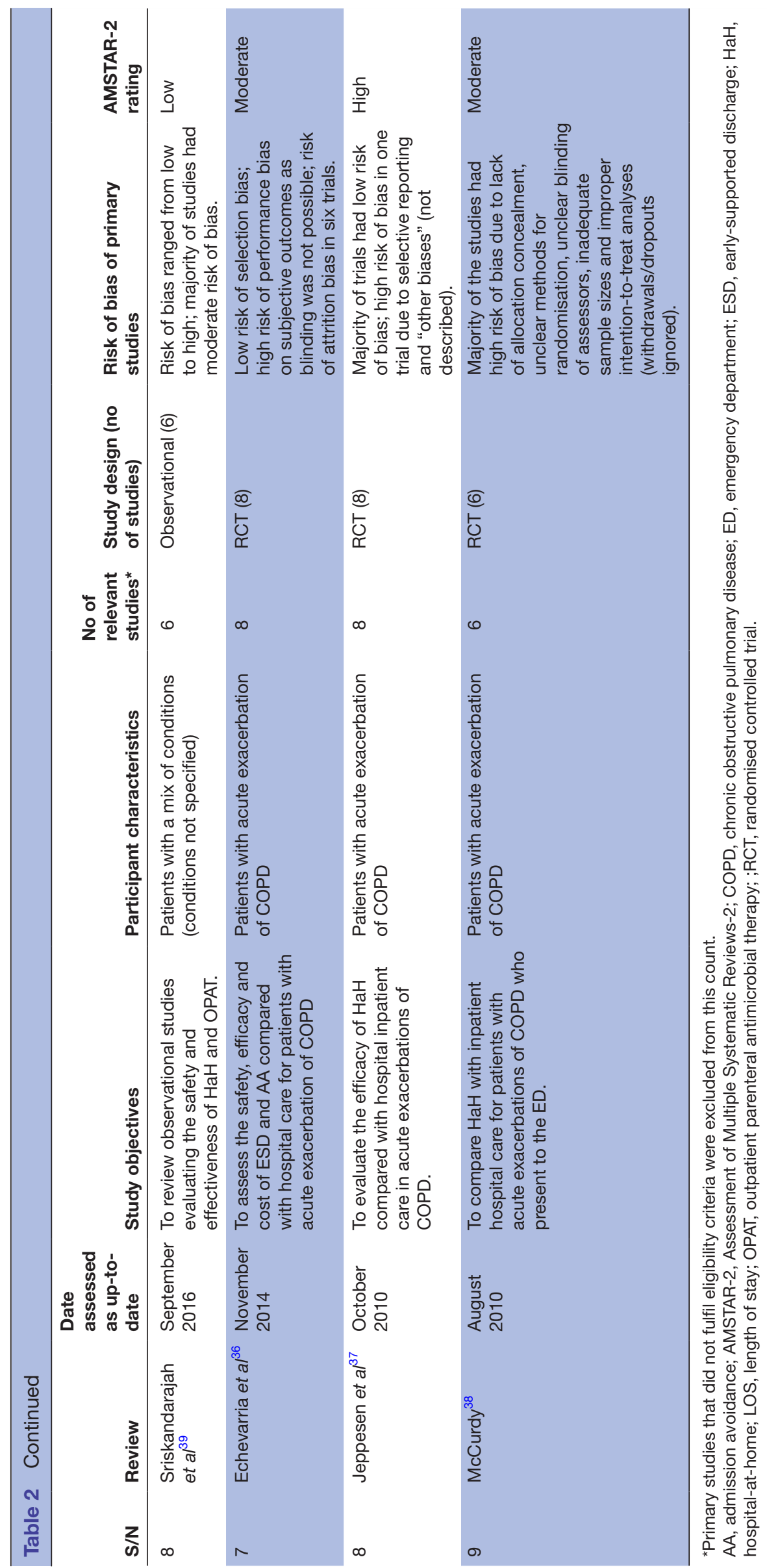




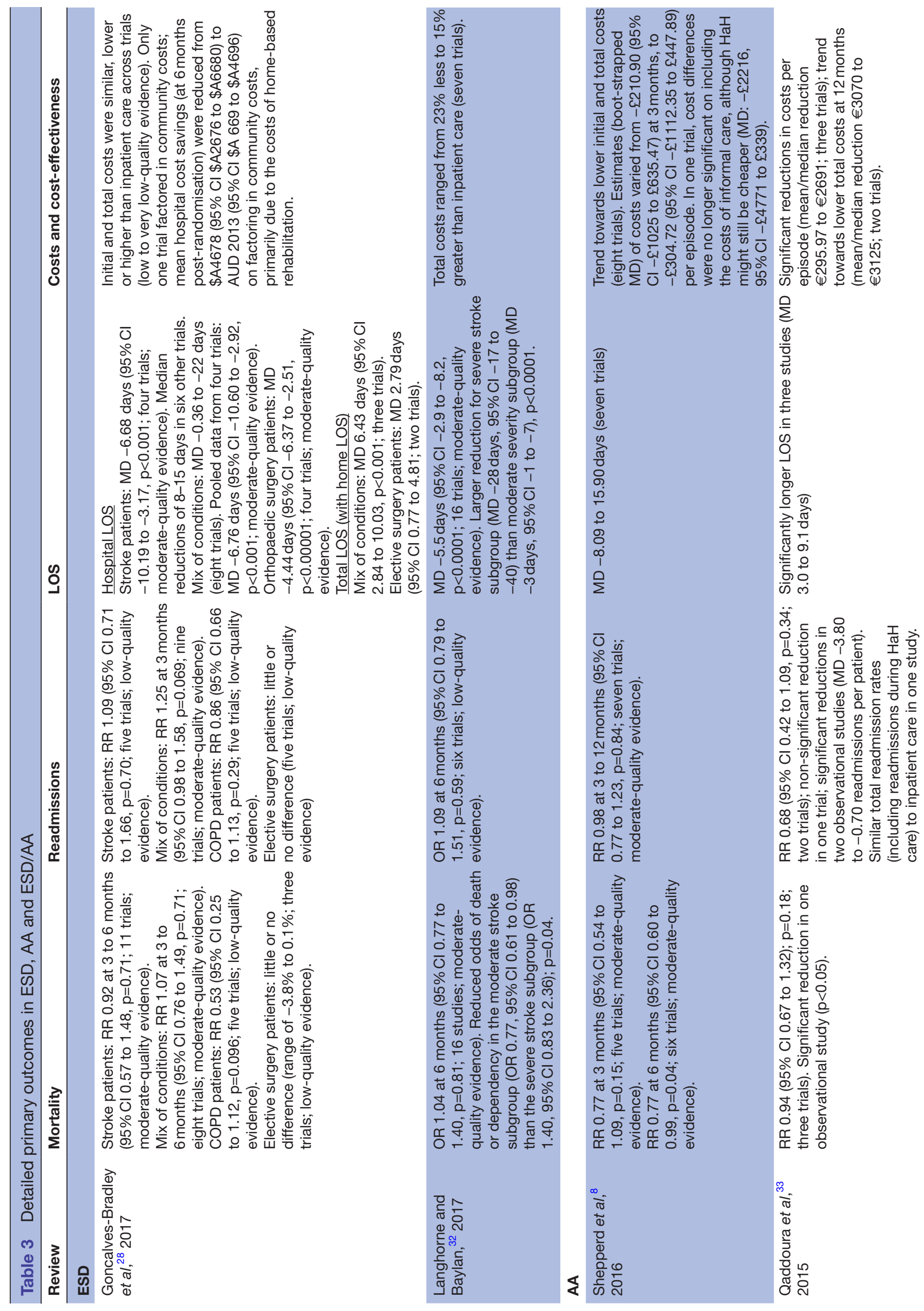

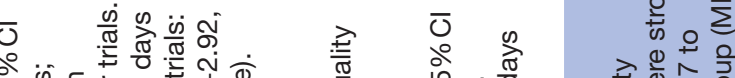

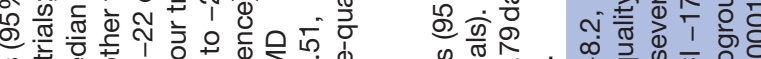
N त्र $\infty$

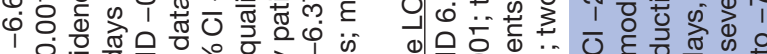
Q v

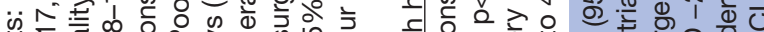

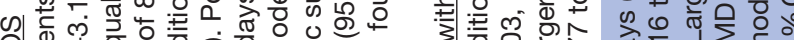

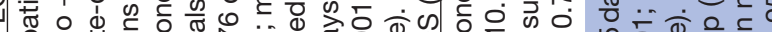

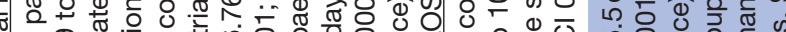

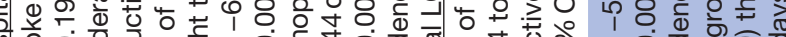

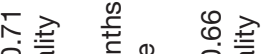

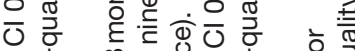

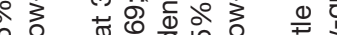

足:

西

每

i

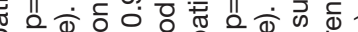

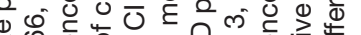

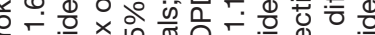

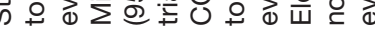

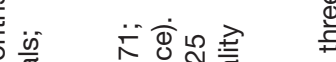

西

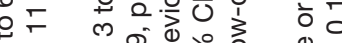

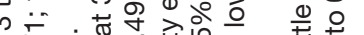

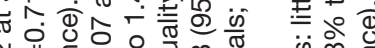

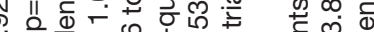

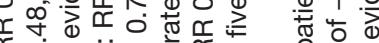

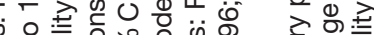

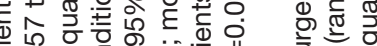

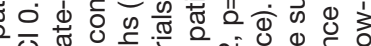

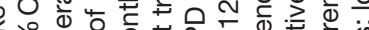

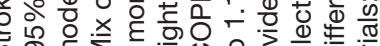

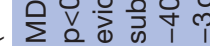

(o

.

일

宊

이잉

近

क्ञ

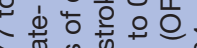

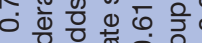

O

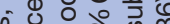

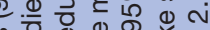

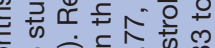

0 ब.

0 -

111 o ट

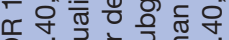




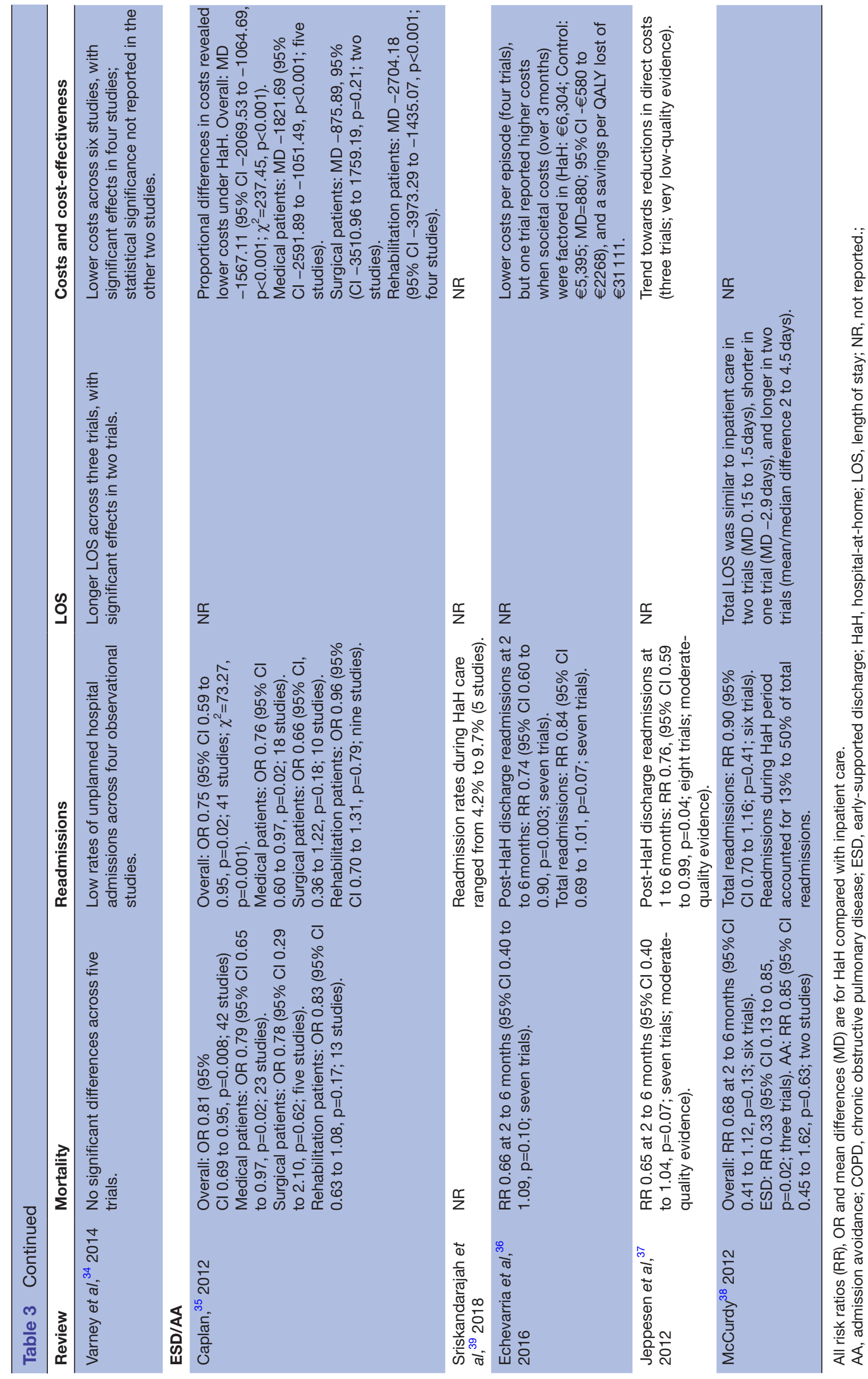


A review for heart failure ${ }^{33}$ observed a trend towards fewer readmissions (post-discharge from $\mathrm{HaH}$ ) under $\mathrm{HaH}$ programmes (RR $0.68,95 \%$ CI 0.42 to 1.09 ). One study reported total readmissions (inclusive of readmissions during $\mathrm{HaH}$ care), which was comparable between AA and inpatient groups. Another review ${ }^{8}$ did not find significant differences in total readmissions between groups (RR $0.98,95 \%$ CI 0.77 to 1.23 ; moderate-quality evidence).

\section{Resource use and costs}

Two reviews ${ }^{33} 34$ reported longer LOS (at home) under $\mathrm{HaH}$ schemes (MD 3.0-9.1 days). Another review ${ }^{8}$ concluded that the effect on LOS was unclear due to high heterogeneity (MD -8.09 to 17 days). However, there was a general trend towards longer LOS.

Three reviews ${ }^{833}$ found that $\mathrm{HaH}$ reduced costs for the acute episode, with this effect reaching significance in several studies (low-quality evidence). With regards to the mean cost savings per episode, one review ${ }^{8}$ reported estimated savings of $£ 304.72$ found in one study (95\% CI -£447.89 to £1112.35), and another review ${ }^{33}$ reported cost savings ranging from $€ 295.97$ to $€ 2691$. However, Shepperd et al suggested that the cost benefit may be offset by the costs of informal care as one trial revealed that the cost reduction was no longer significant on including informal care costs. Two reviews ${ }^{833}$ revealed a trend towards lower total costs (inclusive of follow-up costs) under $\mathrm{HaH}$, although Qaddoura $e t a l^{33}$ concluded that the effect was unclear as few studies included indirect costs.

\section{Patient and caregiver outcomes}

One review ${ }^{8}$ observed significantly higher levels of patient satisfaction in $\mathrm{HaH}$ than inpatient care across most trials (low-quality evidence). Two other reviews ${ }^{33} 34$ also found high levels of satisfaction among $\mathrm{HaH}$ patients in observational studies. For caregiver outcomes, Shepperd $e t a l^{8}$ concluded that there was insufficient evidence as two trials revealed conflicting results.

\section{Process indicators}

One review ${ }^{8}$ reported that recruitment rates for $\mathrm{HaH}$ were low but no data was provided. One review ${ }^{34}$ concluded that $\mathrm{HaH}$ generally did not affect the rate of adverse events. Shepperd $e t a l^{8}$ reported a lack of difference in clinical complications in one trial, and significantly lower rates of bowel and urinary complications and behavioural problems (in dementia patients) under $\mathrm{HaH}$ in two trials.

\section{Early-supported discharge and admission avoidance Clinical outcomes}

One review ${ }^{35}$ for a mix of conditions reported significantly lower mortality under $\mathrm{HaH}$ as compared with inpatient care (OR 0.81, 95\% CI 0.69 to 0.95 ). However, this analysis should be interpreted with caution as several of the included primary studies ${ }^{40-43}$ did not meet the criteria of $\mathrm{HaH}$ (eg, nature of care was long-term care or active care was not provided by healthcare professionals). Three reviews for patients with chronic obstructive pulmonary disease (COPD) $)^{36-38}$ reported a trend towards lower mortality under $\mathrm{HaH}$ (RR 0.65 to 0.68 ; moderate-quality evidence).

Caplan ${ }^{35}$ reported that $\mathrm{HaH}$ significantly reduced readmissions compared with inpatient care (OR 0.75, 95\% CI 0.59 to 0.95$)$. However, interpretation of this result is constrained as the analysis included four irrelevant studies (psychiatric studies), and this subgroup had the largest effect size among the various specialties. It is also unclear if readmissions during $\mathrm{HaH}$ care were included in these analyses. For patients with COPD, two reviews ${ }^{3637}$ found significant reductions in post-HaH discharge readmissions (RR 0.74-0.76; moderate-quality evidence), while two reviews ${ }^{36} 38$ observed non-significant reductions in total readmissions (RR 0.84-0.90).

\section{Resource use and costs}

One review ${ }^{38}$ concluded that the effect of $\mathrm{HaH}$ on total LOS for patients with COPD could not be determined due to conflicting findings.

Caplan ${ }^{35}$ reported that $\mathrm{HaH}$ significantly reduced costs compared with inpatient care, although with significant heterogeneity. The cost components of these analyses were unclear. Two reviews for patients with COPD $^{36} 37$ concluded that evidence of cost savings under $\mathrm{HaH}$ was of very low quality due to heterogeneity. Echevarria $e t a l^{36}$ reported that one study observed higher costs for $\mathrm{HaH}$ than inpatient care when societal costs were included. The study also found that $\mathrm{HaH}$ was associated with a savings per quality-adjusted life year (QALY) lost of €31 111.

\section{Patient and caregiver outcomes}

One review ${ }^{35}$ reported that majority of the trials revealed higher patient and caregiver satisfaction, and lower caregiver burden under $\mathrm{HaH}$. Two reviews ${ }^{37}{ }^{38}$ reported that the effect of $\mathrm{HaH}$ on patient satisfaction for patients with COPD was inconclusive due to limited data (low-quality evidence). One trial observed that $\mathrm{HaH}$ did not affect caregiver satisfaction. ${ }^{37}$

\section{Process indicators}

COPD-specific reviews ${ }^{36-38}$ reported low recruitment rates, with the percentage of eligible patients ranging from $11 \%$ to $39 \%$ across trials. A total of $56 \%-88.5 \%$ of eligible patients consented to participation. ${ }^{38}$

McCurdy ${ }^{38}$ concluded that $\mathrm{HaH}$ did not affect the rate of clinical complications, although there were lower rates of urinary tract infections under $\mathrm{HaH}$ in one trial. A review of observational studies ${ }^{39}$ observed higher rates of complications in the $\mathrm{HaH}$ group than the inpatient group in one study, but the $\mathrm{HaH}$ group was also seven times larger. In another study, patients receiving intravenous antibiotics under AA were at lower risk of adverse effects than ESD patients.

\section{Facilitators and barriers}

Facilitators to implementation include coordinated and multidisciplinary $\mathrm{HaH}$ teams. ${ }^{8}{ }^{32}$ Barriers to 
implementation include physical distance of patients' homes to the hospital ${ }^{3738}$ medical condition stability ${ }^{83738}$ and level of disability. ${ }^{28}{ }^{32}$ ESD led to greater reductions in death or dependency ${ }^{32}$ and higher cost-effectiveness in one trial ${ }^{28}$ when limited to patients with mild or moderate disability. In addition, caregiver availability was an eligibility criteria in one-third to half of the studies across reviews. ${ }^{82}$

\section{DISCUSSION}

Across ESD, AA and ESD/AA reviews, $\mathrm{HaH}$ was generally associated with similar or improved outcomes for mortality and readmissions, shorter hospital LOS and high levels of patient satisfaction compared with inpatient care. While there was a trend towards increased total LOS under both ESD and AA, this is possibly due to the inclusion of postacute care in some schemes. ${ }^{44}{ }^{45}$ In traditional hospitalisations, patients would likely have been transferred to step-down care facilities (eg, community hospitals, skilled nursing facilities) which are not factored into LOS calculations.

In comparing ESD and AA reviews, ESD has been better studied, but AA may lead to greater benefits in terms of clinical outcomes and costs. AA programmes resulted in either comparable or lower mortality and readmission rates when compared with inpatient care. On the other hand, mortality and readmission rates under ESD were generally similar to inpatient care, but with a concerning trend of higher readmissions in schemes for a mix of conditions. The observed benefit of AA over ESD could have been driven by reductions in iatrogenic events as suggested by past research. ${ }^{345}$ However, limited reporting on adverse events precluded clear conclusions. In addition, although the quality of evidence for costs was low across reviews, AA studies revealed consistent cost benefits, in contrast to inconclusive findings under ESD. These observations are aligned with propositions that AA may result in greater cost savings than ESD due to the complete substitution of hospital stay. ${ }^{34}$

Reviews that included both ESD and AA studies were mainly COPD-specific reviews which revealed evidence for lower mortality and readmissions, although with unclear evidence for resource use. Among these reviews, one review ${ }^{38}$ conducted subgroup analyses on clinical outcomes by programme type, but the small number of studies $(n=5)$ precluded conclusive interpretation. It should be noted that one review ${ }^{35}$ conducted subgroup analyses by the degree of substitution of hospital stay (more than or less than 50\%), but the $50 \%$ criterion employed did not adequately capture the distinction between ESD and AA. ESD/AA reviews with planned subgroup analyses for programme type should continue to be explored in future research.

Based on the existing evidence, it is recommended to prioritise AA over ESD in $\mathrm{HaH}$ implementations due to potential benefits in terms of clinical outcomes, costs and the complete substitution of hospital stay under AA. Nonetheless, it should be cautioned that the strength of evidence for our recommendation is low due to the limitations of our indirect and post hoc comparisons. This is especially so given the heterogeneity in implementation and patient groups across studies. We also acknowledge that it may not always be possible to maintain a clear distinction between ESD and AA in real-life implementations due to operational considerations.

\section{Recommendations for practice}

The encouraging evidence for clinical outcomes, LOS and costs under $\mathrm{HaH}$, especially the AA model, highlights an opportunity to tackle the bed shortages and spiralling costs faced by health systems. This is particularly pertinent to advanced economies which are grappling with ageing populations and an increasing burden of multimorbid, complex cases. Moreover, the current COVID-19 pandemic provides greater impetus for $\mathrm{HaH}$ implementation. These schemes can be pivotal in decentralising care, facilitating rapid ramp-ups in bed capacity, ${ }^{46}$ and controlling nosocomial infections. ${ }^{47}$

Notwithstanding the strengths of $\mathrm{HaH}$, the inherent complexity of $\mathrm{HaH}$ poses implementation challenges ${ }^{48}$ and several factors should be deliberated when planning such schemes. At a broad level, contextual factors for consideration include hospital location and payment structures. Given the low recruitment rates in $\mathrm{HaH}$ schemes, HaH may be more practical in urban areas with a sufficient and predictable patient load to allow for greater economies of scale. ${ }^{1749} 50$ Second, many countries where $\mathrm{HaH}$ has been widely implemented (eg, Australia, UK) have single-payer systems and strong imperatives to keep medical costs low, whereas greater barriers are evident in countries such as the US where payment norms are still predominantly episodic and fee-for-service. ${ }^{51}$ Nonetheless, with the shift towards value-based payments ${ }^{52}$ and integrated care ${ }^{53}$ across health systems, a more conducive environment for $\mathrm{HaH}$ may be emerging.

At the programme level, care enablers include the proximity of patients' homes to the hospital and suitability of home settings. With the policy shift towards bringing care closer to the community, especially in advanced economies ${ }^{54}$ it may be timely to re-examine how future urban planning and housing designs can support models of care such as $\mathrm{HaH}$. Care enablers identified in primary studies which were not included in the current paper deserve mention as well. These include skills training of staff to provide care in $\mathrm{HaH}$ schemes ${ }^{55} 56$ and assistive technologies such as telemedicine. Regarding the latter, a recent trial ${ }^{57}$ demonstrated the safety and effectiveness of a virtual $\mathrm{HaH}$ programme that exclusively utilised telehealth during COVID-19.

Several reviews highlighted that $\mathrm{HaH}$ may not be suitable for patients with intensive care needs, or who do not live with caregivers. However, a possible concern with necessitating caregiver availability is the transfer of burden of care from hospitals to family caregivers. In fact, by incorporating appropriate safeguards (eg, panic buttons, fall-detection sensors), caregiver reliance can be reduced while ensuring 
that patients receive quality care. Hence, moving beyond the traditional model of reliance on care providers, $\mathrm{HaH}$ can serve as an avenue for patient empowerment by increasing patient involvement in their own care and boosting their confidence in self-management. By extending $\mathrm{HaH}$ eligibility to patients without caregivers, more will stand to benefit from $\mathrm{HaH}$ schemes as well.

\section{Evidence gaps}

The most well-studied diagnostic groups were COPD and stroke, with evidence of positive clinical outcomes for patients with COPD. Past research has indicated that other conditions are also suitable for $\mathrm{HaH}$, such as cellulitis and deep-vein thrombosis. ${ }^{58}{ }^{59}$ However, there was a lack of subgroup analyses or reviews for these conditions, hence the effects of $\mathrm{HaH}$ for these groups require further clarification.

It was unclear in multiple reviews if early readmissions during $\mathrm{HaH}$ care were factored into analyses for readmissions, hence it was challenging to make comparisons across reviews. Future reviews should clearly report both early and total readmissions. The evidence on costs was of low quality due to heterogeneity across studies, and few studies assessed cost-effectiveness. Future studies could consider full economic evaluations that include all direct and indirect costs (eg, informal care costs) to better guide policy and decision making. Further clarification on caregiver outcomes, especially caregiver burden, is also required given caregivers' crucial roles in $\mathrm{HaH}$ programmes. ${ }^{21}$

Lastly, there was limited reporting on facilitators, barriers and process indicators such as adverse events. Future trials and reviews should report these given that one of the initial objectives of developing the $\mathrm{HaH}$ model was to reduce iatrogenic events, and the incidence of iatrogenic events is also a frequently theorised comparison point between ESD and AA.

\section{Limitations}

The first limitation is the lack of a registered protocol for this study. In addition, the current research might have missed primary studies that were not included in the systematic reviews, and we also excluded grey literature. However, we attempted to address these limitations by highlighting relevant findings from primary studies and government publications.

Outcomes were mainly limited by small sample sizes and heterogeneous measurement tools in the primary studies. Also, two reviews ${ }^{32} 35$ included primary studies that did not meet the criteria of $\mathrm{HaH}$, hence there were likely inaccuracies in the reported effect estimates.

Lastly, overlaps in primary studies across reviews could have inflated results. This limitation was most pronounced for COPD-specific reviews (90\% overlap), but these reviews were included as they provided insight on different outcomes.

\section{CONCLUSION}

$\mathrm{HaH}$ is largely safe and effective for suitable patients requiring hospital-level care. $\mathrm{HaH}$ generally leads to similar or improved clinical outcomes, shorter hospital LOS and high patient satisfaction when compared with inpatient care. HaH particularly warrants greater attention in health systems that are challenged by capacity constraints and rising costs. Moreover, based on preliminary comparisons, AA schemes should be prioritised in further explorations of $\mathrm{HaH}$ due to the complete substitution of hospital stay, and possible cost savings which will likely increase with scaled up programmes. However, for both AA and ESD, several outcomes and process indicators still require further clarification, such as caregiver outcomes, cost-effectiveness and clinical complications.

Contributors As the corresponding author, MQL contributed to the design, data acquisition, analysis and interpretation of the findings, as well as drafting and revising the article. CWL and YFL made a substantial contribution to the conception and design of the study, as well as to interpreting findings, outlining and critical revising of the article. All the authors have provided final approval of the submitted manuscript.

Funding The authors have not declared a specific grant for this research from any funding agency in the public, commercial or not-for-profit sectors.

Competing interests None declared.

Patient consent for publication Not required.

Provenance and peer review Not commissioned; externally peer reviewed.

Data availability statement Data sharing not applicable as no datasets generated and/or analysed for this study. All data were obtained from published studies.

Supplemental material This content has been supplied by the author(s). It has not been vetted by BMJ Publishing Group Limited (BMJ) and may not have been peer-reviewed. Any opinions or recommendations discussed are solely those of the author(s) and are not endorsed by BMJ. BMJ disclaims all liability and responsibility arising from any reliance placed on the content. Where the content includes any translated material, BMJ does not warrant the accuracy and reliability of the translations (including but not limited to local regulations, clinical guidelines, terminology, drug names and drug dosages), and is not responsible for any error and/or omissions arising from translation and adaptation or otherwise.

Open access This is an open access article distributed in accordance with the Creative Commons Attribution Non Commercial (CC BY-NC 4.0) license, which permits others to distribute, remix, adapt, build upon this work non-commercially, and license their derivative works on different terms, provided the original work is properly cited, appropriate credit is given, any changes made indicated, and the use is non-commercial. See: http://creativecommons.org/licenses/by-nc/4.0/.

ORCID iDs

Man Qing Leong http://orcid.org/0000-0002-6299-1685

Yi Feng Lai http://orcid.org/0000-0002-1318-3032

\section{REFERENCES}

1 de Sousa Vale J, Franco Al, Oliveira CV. Hospital at home: an overview of literature. Home Health Care Manage Pract 2019.

2 Levi B, Borow M, Wapner L, et al. Home hospitalization worldwide and in Israel. Isr Med Assoc J 2019;21:565-7.

3 Cunningham P, Sammut J. Inadequate acute hospital beds and the limits of primary care and prevention. Emerg Med Australas 2012;24:566-72.

4 Sprivulis PC, Da Silva J-A, Jacobs IG, et al. The association between hospital overcrowding and mortality among patients admitted via Western Australian emergency departments. Med J Aust 2006;184:208-12.

5 Richardson DB. Increase in patient mortality at 10 days associated with emergency department overcrowding. Med J Aust 2006;184:213-6.

6 Kirby A, Kjesbo A. Tapping into hidden Hospital bed capacity. Healthcare Financial Management, 2003: 38.

7 Sambamoorthi U, Tan X, Deb A. Multiple chronic conditions and healthcare costs among adults. Expert Rev Pharmacoecon Outcomes Res 2015;15:823-32.

8 Shepperd S, lliffe S, Doll HA, et al. Admission avoidance Hospital at home. Cochrane Database Syst Rev 2016;9:Cd007491. 
9 Leff B, Burton L, Mader SL, et al. Hospital at home: feasibility and outcomes of a program to provide hospital-level care at home for acutely ill older patients. Ann Intern Med 2005;143:798-808.

10 Ram FSF, Wedzicha JA, Wright J, et al. Hospital at home for patients with acute exacerbations of chronic obstructive pulmonary disease: systematic review of evidence. BMJ 2004;329:315.

11 Summerfelt WT, Sulo S, Robinson A, et al. Scalable Hospital at home with virtual physician visits: pilot study. Am J Manag Care 2015;21:675-84.

12 Leff B. Defining and disseminating the hospital-at-home model. CMAJ 2009;180:156-7.

13 Caplan G. Does 'Hospital in the Home' treatment prevent delirium? Aging health 2008;4:69-74.

14 Cryer L, Shannon SB, Van Amsterdam M, et al. Costs for 'hospital at home' patients were 19 percent lower, with equal or better outcomes compared to similar inpatients. Health Aff 2012;31:1237-43.

15 Aimonino Ricauda N, Tibaldi V, Leff B, et al. Substitutive "hospital at home" versus inpatient care for elderly patients with exacerbations of chronic obstructive pulmonary disease: a prospective randomized, controlled trial. J Am Geriatr Soc 2008;56:493-500.

16 Prothero L, Barley E, Galloway J, et al. The evidence base for psychological interventions for rheumatoid arthritis: a systematic review of reviews. Int J Nurs Stud 2018;82:20-9.

17 National Institute for Health and Care Excellence. Chapter 12 alternatives to hospital care (NICE guideline 82), 2017.

18 Mader SL, Medcraft MC, Joseph C, et al. Program at home: a Veterans Affairs healthcare program to deliver hospital care in the home. J Am Geriatr Soc 2008;56:2317-22.

19 Maclntyre CR, Ruth D, Ansari Z. Hospital in the home is cost saving for appropriately selected patients: a comparison with in-hospital care. Int J Qual Health Care 2002;14:285-93.

20 Anderson C, Rubenach S, Mhurchu CN, et al. Home or hospital for stroke rehabilitation? Results of a randomized controlled trial. Stroke 2000;31:1024-31.

21 Rossinot $\mathrm{H}$, Marquestaut $\mathrm{O}$, de Stampa M. The experience of patients and family caregivers during hospital-at-home in France. BMC Health Serv Res 2019;19:470-70.

22 Luckett T, Davidson PM, Lam L, et al. Do community specialist palliative care services that provide home nursing increase rates of home death for people with life-limiting illnesses? A systematic review and meta-analysis of comparative studies. $J$ Pain Symptom Manage 2013:45:279-97.

23 Richards SH, Coast J, Gunnell DJ, et al. Randomised controlled trial comparing effectiveness and acceptability of an early discharge, Hospital at home scheme with acute hospital care. BMJ 1998;316:1796-801.

24 Shepperd S, Harwood D, Jenkinson C, et al. Randomised controlled trial comparing Hospital at home care with inpatient hospital care. I: three month follow up of health outcomes. BMJ 1998;316:1786-91.

25 Crotty M, Whitehead CH, Gray S, et al. Early discharge and home rehabilitation after hip fracture achieves functional improvements: a randomized controlled trial. Clin Rehabil 2002;16:406-13.

26 Hill SP, Flynn J, Crawford EJP. Early discharge following total knee replacement - a trial of patient satisfaction and outcomes using an orthopaedic outreach team. J Orthopaedic Nurs 2000;4:121-6.

27 Caplan GA, Coconis J, Board N, et al. Does home treatment affect delirium? a randomised controlled trial of rehabilitation of elderly and care at home or usual treatment (the REACH-OUT trial). Age Ageing 2006;35:53-60.

28 Gonçalves-Bradley DC, lliffe S, Doll HA, et al. Early discharge Hospital at home. Cochrane Database Syst Rev 2017:6:Cd000356.

29 Mainz J. Defining and classifying clinical indicators for quality improvement. Int J Qual Health Care 2003:15:523-30.

30 Shea BJ, Reeves BC, Wells G, et al. AMSTAR 2: a critical appraisal tool for systematic reviews that include randomised or nonrandomised studies of healthcare interventions, or both. $B M J$ 2017;358:j4008.

31 Pollock M, Fernandes RM, Becker LA. Chapter V: Overviews of Reviews. In: Cochrane Handbook for systematic reviews of interventions version 6.1 (updated September 2020). Cochrane, 2020.

32 Langhorne P, Baylan S, Early Supported Discharge Trialists. Early supported discharge services for people with acute stroke. Cochrane Database Syst Rev 2017;7:Cd000443.

33 Qaddoura A, Yazdan-Ashoori P, Kabali C, et al. Efficacy of hospital at home in patients with heart failure: a systematic review and metaanalysis. PLoS One 2015;10:e0129282.

34 Varney J, Weiland TJ, Jelinek G. Efficacy of hospital in the home services providing care for patients admitted from emergency departments: an integrative review. Int J Evid Based Healthc 2014:12:128-41.
35 Caplan GA. A meta-analysis of "hospital in the home". Med J Aust 2013:198:195-6.

36 Echevarria C, Brewin K, Horobin $\mathrm{H}$, et al. Early supported Discharge/Hospital at home for acute exacerbation of chronic obstructive pulmonary disease: a review and meta-analysis. COPD 2016;13:523-33.

37 Jeppesen E, Brurberg KG, Vist GE, et al. Hospital at home for acute exacerbations of chronic obstructive pulmonary disease. Cochrane Database Syst Rev 2012:Cd003573.

38 McCurdy BR. Hospital-at-home programs for patients with acute exacerbations of chronic obstructive pulmonary disease (COPD): an evidence-based analysis. Ont Health Technol Assess Ser 2012:12:1-65.

39 Sriskandarajah S, Hobbs J, Roughead E, et al. Safety and effectiveness of 'hospital in the home' and 'outpatient parenteral antimicrobial therapy' in different age groups: A systematic review of observational studies. Int J Clin Pract 2018:e13216.

40 Melin AL, Bygren LO. Efficacy of the rehabilitation of elderly primary health care patients after short-stay hospital treatment. Med Care 1992;30:1004-15.

41 Bonnema J, van Wersch AM, van Geel AN, et al. Medical and psychosocial effects of early discharge after surgery for breast cancer: randomised trial. BMJ 1998;316:1267-71.

42 Levine M, Gent M, Hirsh J, et al. A comparison of low-molecularweight heparin administered primarily at home with unfractionated heparin administered in the hospital for proximal deep-vein thrombosis. N Engl J Med 1996;334:677-81.

43 Koopman MM, Prandoni P, Piovella F, et al. Treatment of venous thrombosis with intravenous unfractionated heparin administered in the hospital as compared with subcutaneous low-molecular-weight heparin administered at home. The Tasman Study Group. N Engl J Med 1996;334:682-7.

44 Tibaldi V, Isaia G, Scarafiotti C, et al. Hospital at home for elderly patients with acute decompensation of chronic heart failure: a prospective randomized controlled trial. Arch Intern Med 2009;169:1569-75.

45 Ricauda NA, Bo M, Molaschi M, et al. Home hospitalization service for acute uncomplicated first ischemic stroke in elderly patients: a randomized trial. J Am Geriatr Soc 2004;52:278-83.

46 Heller DJ, Ornstein KA, DeCherrie LV, et al. Adapting a Hospital-atHome care model to respond to new York City's COVID-19 crisis. $J$ Am Geriatr Soc 2020;68:1915-6.

47 Coloma E, Nicolás D. Hospital at home units in the post-COVID 19 era. Eur J Clin Invest 2020;50:e13390.

48 Sitammagari K, Murphy S, Kowalkowski M, et al. Insights From Rapid Deployment of a "Virtual Hospital" as Standard Care During the COVID-19 Pandemic. Ann Intern Med 2020:M20-4076.

49 Saenger P, Federman AD, DeCherrie LV. Choosing inpatient vs home treatment: why patients accept or decline Hospital at home. J Am Geriatr Soc 2020

50 Klein S, Hostetter M, McCarthy D. The hospital at home model: bringing hospital-level care to the patient the Commonwealth fund, 2016. Available: https://www.commonwealthfund.org/publications/ case-study/2016/aug/hospital-home-model-bringing-hospital-levelcare-patient\#/

51 Marsteller JA, Burton L, Mader SL, et al. Health care provider evaluation of a substitutive model of hospital at home. Med Care 2009:47:979-85.

52 Eijkenaar F, Emmert M, Scheppach M, et al. Effects of pay for performance in health care: a systematic review of systematic reviews. Health Policy 2013;110:115-30.

53 Nurjono M, Yoong J, Yap P, et al. Implementation of integrated care in Singapore: a complex adaptive system perspective. Int $J$ Integr Care 2018;18:4.

54 Khalik S. The'3 Beyonds': Singapore's strategy to sustain quality healthcare as demand rises. 30. The Straits Times, 2017.

55 Brody AA, Arbaje Al, DeCherrie LV, et al. Starting up a hospital at home program: facilitators and barriers to implementation. J Am Geriatr Soc 2019;67:588-95.

56 Dismore LL, Echevarria C, van Wersch A, et al. What are the positive drivers and potential barriers to implementation of hospital at home selected by low-risk DECAF score in the UK: a qualitative study embedded within a randomised controlled trial. BMJ Open 2019:9:e026609.

57 Levine DM, Ouchi K, Blanchfield B, et al. Hospital-Level care at home for acutely ill adults. Ann Intern Med 2020;172:77-85.

58 Wilson SF, Collins N. Hospital in the home: what next? Med J Aust 2006;184:141-2.

59 NSW Ministry of Health. Adult and paediatric hospital in the home guideline, 2018. 